cause from all we know we would expect a somewhat lesser rate of solvent denudation as the world gets older and the land gets more and more loaded with the washedout materials of the rocks.

Both the methods referred to of finding the age assume the principle of uniformity. The geologist contends for uniformity throughout the past physical history of the earth. The physicist claims the like for the change rates of the radioactive elements. Now the study of the rocks enables us to infer something as to the past history of our globe. Nothing is, on the other hand, known respecting the origin of uranium or thorium, the parent radioactive bodies. And while not questioning the law and regularity which undoubtedly prevail in the periods of the members of the radioactive families, it appears to me that it is allowable to ask if the change rate of uranium has been always what we now believe it to be. This comes to much the same thing as supposing that atoms possessing a faster change rate once were associated with it which were capable of yielding both helium and lead to the rocks. Such atoms might have been collateral in origin with uranium from some antecedent element. Like helium, lead may be a derivative from more than one sequence of radioactive changes. In the present state of our knowledge the possibilities are many. The change rate is known to be connected with the range of the alpha ray expelled by the transforming element; and the conformity of the halo with our existing knowledge of the ranges is reason for assuming that, whatever the origin of the more active associate of uranium, this passed through similar elemental changes in the progress of its disintegration. There may, however, have been differences in the ranges which the halo would not reveal. It is remarkable that uranium at the present time is apparently responsible for two

\section{Cost of Ice Making in Small Plants*} By R. P. Kehoe

The business of ice manufacture is sometimes disparaged and manufacturers of such machinery are criticized for the apparent failure of a few plants. The cause is usually attributed to inefficient equipment or misleading statements on the part of the builders. misleading statements on the part of the builders.
Occasionally the purchaser is convinced that the plant is properly constructed, but believes his operators to be at fault.

In almost every instance the lack of success arises from local conditions which the most perfect plant could not overcome. This is especially true in installations of small capacity. Large plants are only installed where there is considerable demand which lasts for several months during each year, but small plants may be installed in localities having little population and requiring ice for only a comparatively short period.

In cities of any size there is a certain demand throughout the year. Hotels, saloons, butchers and the like require ice even in the coldest weather. Inhabitants of cities are also accustomed to use ice constantly. But in suburbs, country towns and villages little or no ice is used during the cooler months. June, July, August and September are practically the only months which can be counted on to produce a maximum demand. Moreover, some days during these months will be cool and the business will fall off. Under such conditions the entire output of a plant may not exceed 30 to 40 the entire output of a plant may
per cent of the full yearly capacity.

If the percentage of yearly output were precisely the same for two plants, one of large and one of small capacity, the manufacturing cost per ton should be and is less in the large plant; but usually the small plant must bear the burden of a low yearly load factor, so that it is actually necessary to make a more careful so that it is actually necessary to make a more careful
investigation of local conditions governing demand and investigation of local conditions governing demand and
selling price in the building of a small plant than a large one.

The daily manufacturing cost when the plant is operating at full capacity gives positively no idea of possible success; such figures are entirely misleading. Many small plants now in operation show little or no profit at the end of each year and the owner cannot understand it when the daily operating cost per t॰n understand it when the daily operating cost per ton
is only about half the selling price. The cost of upkeep, depreciation and labor during shutdowns and comparatively low yearly consumption are not considered.

Very often in a small business the cost of delivery and manufacturing are confused, and the fact that a loss in one end eats up part or all of the profit in the other may never be discovered. The average owner of a small ice plant is not often an expert business man and cannot analyze the combination of manufacturing and delivery of ice with much accuracy.

The accompanying tabulation clearly shows how much the yearly consumption will affect the total cost of production. The figures covering labor and fuel represent an average and can be adjusted to suit any special locality, but the comparison is a true one in any case.

Reproduced from Pure Products. alpha rays of very different ranges. If these proceed from different elements, one should be faster in it change rate than the other. Some guidance may yet be forthcoming from the study of the more obscure problems of radioactivity.

Now it is not improbable that the halo may con tribute directly to this discussion. We can evidently attack the biotite with a known number of alpha ray and determine how many are required to produce certain intensity of darkening, corresponding to that of a halo with a nucleus of measurable dimensions. On certain assumptions, which are correct within defined limits, we can calculate, as I have done above, the number of rays concerned in forming the halo. In doing so we assume some value for the age of the halo. Let us take the maximum radioactive value. A halo originating in Devonian times may attain a certain central blackening from the effects of, say, $10^{8}$ rays But now suppose we find that we cannot produce the same degree of blackening with this number of rays applied in the laboratory. What are we to conclude? I think there is only the one conclusion open to us, that some other source of alpha rays, or a faster rate of supply, existed in the past. And this conclusion would explain the absence of halos from the younger rocks; which, in view of the vast range of effects possible in the development of halos, is, otherwise, not easy to account for. It is apparent that the experiment on the biotite has a direct bearing on the validity of the radioactive method of estimating the age of the rocks. It is now being carried

Finally, there is one very certain and valuable fact to be learned from the halo. The halo has established the extreme rarity of radioactivity as an atomic phe- nomenon. One and all of the speculations as to the slow breakdown of the commoner elements may be dismissed. The halo shows that the mica of the rocks is radioactively sensitive. The fundamental criterion of radioactive change is the expulsion of the alpha ray. The molecular system of the mica and of many other minerals is unstable in presence of these rays, just as a photographic plate is unstable in presence of light. Moreover, the mineral integrates the radioactive effects in the same way as a photographic salt integrates the effects of light. In both cases the feeblest activities become ultimately apparent to our inspection. We have seen that one ray in each year since the Devonian other appearance in the rocks. And we have been able to allocate all the halos so far investigated to one or to allocate all the halos so far investigated to one or
the other of the known radioactive families. We are the other of the known radioactive families. We are been radioactive we must either find characteristic halos produced by them, or else find a complete darkening of the mica. The feeblest alpha rays emitted by the relatively enormous quantities of the prevailing elements, acting over the whole duration of geological time, and it must be remembered that the halos we have been studying are comparatively young, must have registered their effects on the very sensitive minerals.

And thus we are safe in concluding that the common elements, and, indeed, many which would be called rare, are possessed of a degree of stability which has preserved them unchanged since the beginning of geological time. Each unaffected flake of mica is thus unassailable proof of a fact which, but for the halo, would probably, have been for ever beyond our cognizance.
In the table figures are given for total yearly production equivalent to three, four, five and six months full capacity. It must be understood that no plant will simply operate for these periods during the year and then shut down. Maximum capacity is usually

can be assumed as a fair basis. On the mechanical equipment 5 per cent depreciation is conservative, because it does not cover any repairs or upkeep. If this 5 per cent is actually set aside as a sinking fund and invested to earn a normal rate of interest it would

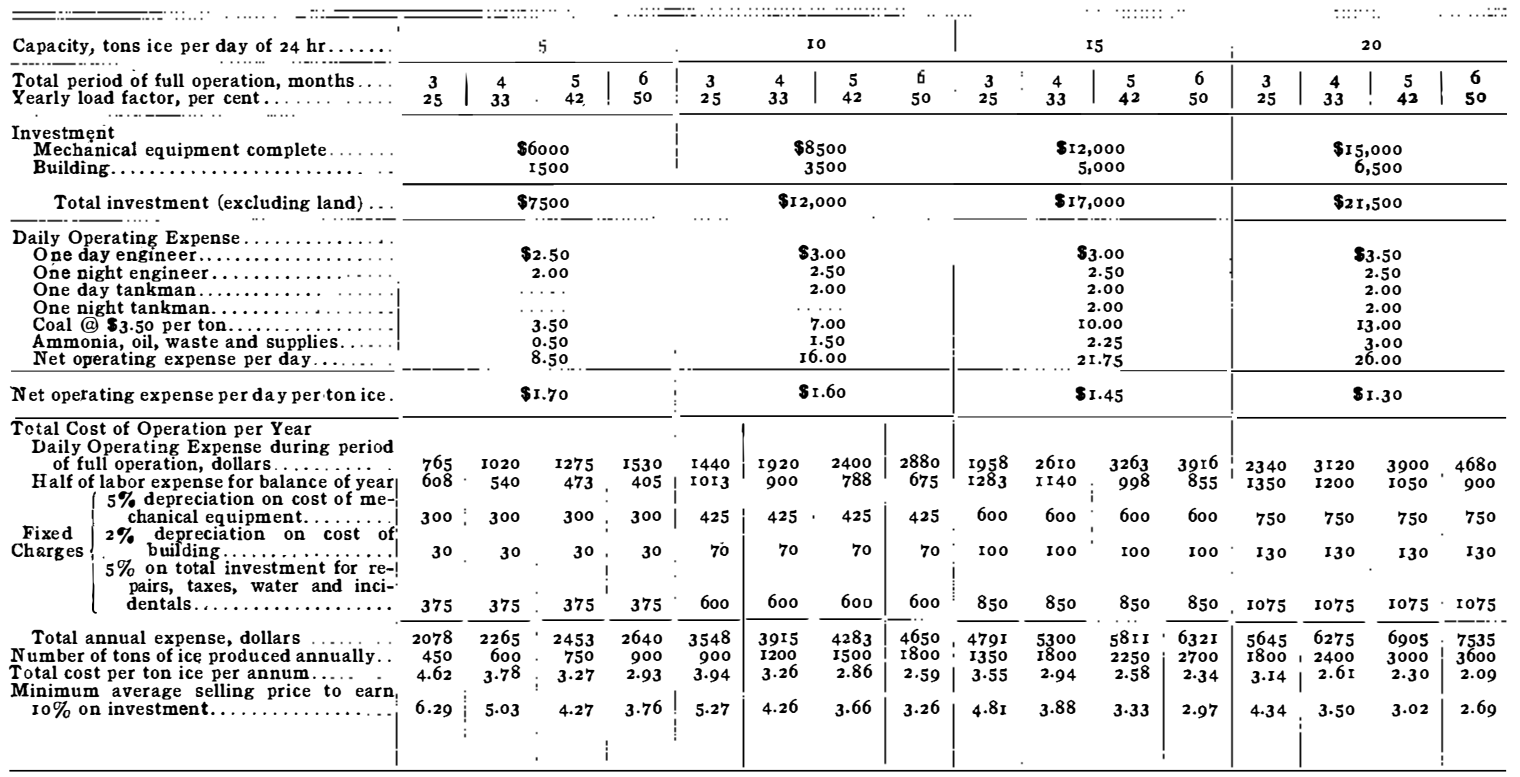

required throughout July and August and possibly part of June and September, but the balance of the time will only demand from 50 per cent down to a very small percentage of the full output. The total amount, however, will in almost every instance equal the full capacity for from three to six months. A plant operating with a yearly load factor of 25 per cent or the equivalent of three months' production may be taken as a bad case, although this is entirely possible in certain localities, particularly in the Northern States or where ice is only required for a summer population. The equivalent of six months' production would, on the plant.

The estimated costs of installation are fairly liberal, but can be depended upon to be very nearly correct. The building for the 5-ton plant is assumed to be of very cheap construction on account of the small size of the plant. The larger plants would need stronger and more elaborate structures, which explains why these figures are not exactly proportional.

In the 5-ton plant the engineers can fire the boiler and also pull the ice. For the 10-ton plant one tankman is figured on to harvest all the ice during the day, as the operation can be arranged in this manner by providing a distilled water-storage tank to collect the condensate during the night. Four men are necessary for both the 15- and 20-ton plants.

During the cooler months the labor expense can be reduced somewhat, and in the tabulation this item is calculated at 50 per cent of the full expense, which equal the total value of the equipment in about 15 years. In addition, 5 per cent on the entire investment is calculated for yearly repairs and incidentals. The cost of property has not been considered and would depend upon the location. This part of the investment really need not be required to earn anything as generally it will increase in value during the life as generally it will increase in value during the
of a plant. It can therefore be considered apart. a plant. It can therefore be considered apart.
The last two lines are of particular interest. It may be noted that the total cost per ton of ice per year ranges from $\$ 2.09$ in the 20 -ton plant with a 50 per cent load factor to $\$ 4.62$ in the 5-ton plant with a 25 per cent load factor. Furthermore, the selling price necessary to obtain to earn 10 per cent on the investment ranges from $\$ 2.69$ to $\$ 6.29$ per ton. These figures may be astonishing to those who only figure on the regular operating expense without regard to the other items, but they are nevertheless perfectly true and represent the only proper basis on which to figure profits.

This article is not intended to discourage investors, for there are hundreds of very profitable plants now in operation that indicate the success of the business in general. The gradual decline of the natural ice trade occasioned by the condemning of questionable sources of supply and the growing preference for the purer artificial product promises a still better outlook in the future. A few failures, however, are often considered to the disadvantage of the entire business and a more universal knowledge of the true circumstances should help the situation. 